\title{
Managing approximation models in multiobjective optimization
}

Y.S. Yang, B.S. Jang, Y.S. Yeun and W.S. Ruy

Struct Multidisc Optim (2002) 24:141-156

DOI 10.1007/s00158-002-0224-0

Unfortunately there were some errors in the authors' names in the original article. All these names are given correctly above.

Published online: 30 June 2003

(C) Springer-Verlag 2003

Y.S. Yang ${ }^{1}$, B.S. Jang ${ }^{1,}$, Y.S. Yeun ${ }^{2}$ and W.S. Ruy ${ }^{1}$

${ }^{1}$ Department of Naval Architecture and Ocean Engineering, Seoul National University, San 56-1, Shillim-dong, Kwanak-gu, Seoul, 151-742, Korea e-mail: ysyang@gong.snu.ac.kr, jbs@insdel.snu.ac.kr, ruy@insdel.snu.ac.kr, beomseon.jang@samsung.com

${ }^{2}$ Department of Mechanical Design Engineering, Deajin University, San 11-1, Sundanri, Pochen, Kyonggi-do, 467-711, Korea

e-mail: yeonyun@road.daejin.ac.kr 